\title{
First-Order Differential Equations with Variable Domains of Piecewise Smooth Operator Coefficients
}

\author{
K. V. Vasilevsky and F. E. Lomovtsev \\ Presented by Academician E.I. Moiseev May 16, 2008
}

Received May 30, 2008

\section{DOI: $10.1134 / \mathrm{S} 1064562408060215$}

This paper describes new results concerning the well-posedness (in the sense of Hadamard) of a firstorder operator-differential equation with piecewise smooth operators defined in variable domains. The operators are not necessarily self-adjoint (in contrast to [1]) and do not necessarily have global smoothing operators with constant domains (in contrast to [2]) or local smoothing operators with variable domains (in contrast to [3]), but they have only majorizing operators with variable domains. For the first time, we prove the smoothness of weak solutions to such equations (Theorem 2). The weak well-posedness of two new mixed problems for variable-order partial differential equations is substantiated.

1. In a Hilbert space $H$ with inner product $(\cdot, \cdot)$ and norm $|\cdot|$, we consider the Cauchy problem

$$
\left.\frac{d u(t)}{d t}+A(t) u=f, \quad t \in\right] 0, T\left[; \quad u(0)=u_{0}\right.
$$

where $u$ and $f$ are $H$-valued functions of $t$ and $A(t)$ denotes linear unbounded operators in $H$ with $t$-dependent domains $D(A(t))$ that satisfy the following conditions.

(I) For any $t \in[0, T]$, the operators $A(t)$ are closed in $H$ and satisfy

$$
\begin{gathered}
{[u]_{(t)}^{2} \equiv \operatorname{Re}\left(A(t) u+c_{0} u, u\right) \geq c_{1}|u|^{2} \forall u \in D(A(t)),} \\
\langle v\rangle_{(t)}^{2} \equiv \operatorname{Re}\left(A^{*}(t) v+c_{0} v, v\right) \geq c_{1}|v|^{2} \\
\forall v \in D\left(A^{*}(t)\right), \quad c_{0} \geq 0, \quad c_{1}>0
\end{gathered}
$$

where $A^{*}(t)$ with domains $D\left(A^{*}(t)\right)$ are the adjoints of $A(t)$ in $H$.

Belarussian State University, pr. Nezavisimosti 4, Minsk, 220050 Belarus

e-mail:phoenix005@rambler.ru,lomovcev@bsu.by
(II) On each partial interval $I_{r}=\left[t_{r}, t_{r+1}\right.$ [ of the partition $\left[0, T\left[=\coprod_{r=0}^{R} I_{r}\right.\right.$, the inverses $A_{0}^{-1}(t) \in \mathscr{B}\left(I_{r}, \mathscr{L}(H)\right)$ of the operators $A_{0}(t)=A(t)+c_{0} I$ are strongly continuous with respect to $t \in I_{r}$ in $H$ and, for almost all (a.a.) $t \in I_{r}$ in $H$, there exists a weak derivative $d A_{0}^{-1}(t) / d t \in$ $L_{\infty}\left(I_{r}, \mathscr{L}(H)\right)$ and

$$
\begin{gathered}
\left|d A_{0}^{-1}(t) / d t g, h\right| \leq c_{2}(r)\left[A_{0}^{-1}(t) g\right]_{(t)}|h| \\
\forall g, h \in H, \quad c_{2}(r) \geq 0, \quad r=0,1, \ldots, R .
\end{gathered}
$$

(III) On each interval $I_{r}(r=0,1, \ldots, R-1)$, the operators $A^{*}(t)$ are subordinated to the adjoints $B^{*}(t)$ (with domains $D\left(B^{*}(t)\right)$ in $H$ ) of some closed operators $B(t)$ with $t$-dependent domains $D(B(t))$.

(a) Conditions I and II hold for the operators $B^{*}(t)$ instead of $A_{0}(t)$ on each interval $I_{r}(r=0,1, \ldots, R-1)$ separately.

(b) The inverses $B^{-1}(t) \in \mathscr{B}\left(I_{r}, \mathscr{L}(H)\right)$ of the operators $B(t)$ are weakly continuous with respect to $t \in I_{r}$ in $H$ and, for a.a. $t \in I_{r}$, they have a weak derivative $\frac{d B^{-1}(t)}{d t} \in L_{\infty}\left(I_{r}, \mathscr{L}(H)\right)$ in $H$ such that

$$
\begin{aligned}
-\operatorname{Re}\left(\varphi,\left(\frac{d B^{-1}(t)}{d t}\right) B(t) \varphi\right) & \leq c_{3}(r)|\varphi|[\operatorname{Re}(B(t) \varphi, \varphi)]^{1 / 2}(5) \\
c_{3} \geq 0, \quad r & =0,1, \ldots, R-1
\end{aligned}
$$

for a.a. $t \in I_{r} \forall \varphi \in D(B(t))$.

(c) At all the discontinuity points $t_{r}$ of $A_{0}^{-1}(t)$,

$$
D\left(A^{*}\left(t_{r}\right)\right) \subset D\left(B^{*}\left(t_{r}-0\right)\right), \quad r=1,2, \ldots, R,
$$

where $D\left(B^{*}\left(t_{r}-0\right)\right)$ are the domains of the left extensions $B^{*}\left(t_{r}-0\right)$ of $B^{*}(t)\left(t<t_{r}\right)$ to the points $t_{r} \in I_{r}$

Condition III(a) implies that the operators $B^{*-1}(t)$ have maximal accretive extensions $B^{*}\left(t_{r}-0\right)$ by conti- 
nuity: for all $g \in H$, we set $v\left(t_{r}\right) \in D\left(B^{*}\left(t_{r}-0\right)\right)$ and $g=$ $B^{*}\left(t_{r}-0\right) v\left(t_{r}\right)$ if $v(t)=B^{*-1}(t) g \rightarrow v\left(t_{r}\right)$ strongly in $H$. Obviously, $B^{*}(t) \mathrm{v}(t)=g \rightarrow g$ strongly in $H$ as $t \hookrightarrow t_{r}, r=$ $1,2, \ldots, R$.

Let $H_{t}^{*-}$ be the antiduals of the Hilbert spaces $H_{t}^{*+}$ obtained as the closure of $D\left(A^{*}(t)\right)$ with respect to the Hermitian norms $\langle\cdot\rangle_{(t)}$ in (3).

Definition 1. A function $u \in \mathcal{H}=L_{2}(] 0, T[, H)$ is called a weak solution to Cauchy problem (1) with a right-hand side $f \in \mathscr{H}^{*-}=L_{2}(] 0, T\left[, H_{t}^{*-}\right)$ and an initial function $u_{0} \in H$ if $u$ satisfies the identity

$$
\begin{aligned}
& \int_{0}^{T}\left\{\left(u(t), A^{*}(t) \varphi(t)\right)-\left(u(t), \frac{d \varphi(t)}{d t}\right)\right\} d t \\
= & \int_{0}^{T}\langle f(t), \varphi(t)\rangle_{(t)} d t+\left(u_{0}, \varphi(0)\right) \forall \varphi \in \Phi .
\end{aligned}
$$

Here, $\Phi=\left\{\varphi \in H: \varphi(t) \in D\left(A^{*}(t)\right), t \in[0, T]\right.$; weak derivative $\left.\frac{d \varphi}{d t}, A^{*}(t) \varphi \in \mathscr{H} ; \varphi(T)=0\right\}$, where $\langle\cdot, \cdot\rangle_{(t)}$ are the antiduality forms between $H_{t}^{*+}$ and $H_{t}^{*-}$.

We analyze the weak well-posedness of Cauchy problem (1) with piecewise smooth unbounded operators and examine the local smoothness of its weak solutions. Additionally, we analyze the well-posedness of previously unstudied mixed problems for partial differential equations with time-discontinuous coefficients in the equations and with piecewise smooth coefficients in the boundary conditions. The existence and uniqueness of weak solutions are stated in Theorem 1; their stability is treated in Corollary 1; their local smoothness, in Corollary 2; and the well-posedness of new mixed problems is stated in Theorems 3 and 4 .

Remark 1. If the operators $A(t)$ satisfy Conditions I and II on $I_{r}$, then $B(t)=A_{0}(t)$ obviously satisfies Condition III(b) on $I_{r}$

2. First, we prove the existence and uniqueness of weak solutions.

Theorem 1. If Conditions I-III are satisfied, then, for any $f \in \mathscr{H}^{*-}$ and $u_{0} \in H$, Cauchy problem (1) has a unique weak solution $u \in \mathcal{H}$.

In [4], Lions' projection theorem [1, p. 37] was used to show that, under Condition I, for any $f \in \mathscr{H}^{*-}$ and $u_{0} \in H$, Cauchy problem (1) has a weak solution $u \in \mathscr{H}$. According to Definition 1, its uniqueness will be proved if we show that the identity

$$
\int_{0}^{T}\left\{\left(u(t), A^{*}(t) \varphi(t)\right)-\left(u(t), \frac{d \varphi(t)}{d t}\right)\right\} d t=0
$$

$\forall \varphi \in \Phi$, which follows from (7) at $f=0$ and $u_{0}=0$, implies that the weak solution $u(t)$ vanishes for a.a. $t$ on each interval $I_{r}(r=0,1, \ldots, R)$ separately.

To show that $u=0$ almost everywhere on $I_{0}=\left[0, t_{1}[\right.$, we set in (8) $\varphi(t)=0$ for $t \in\left[t_{1}, T[\right.$ and $\varphi(t)=$ $A_{0}^{*^{-1}}(t) w(t)$ for $t \in\left[0, t_{1}\left[\right.\right.$, where $w(t)=-\int_{t}^{t_{1}} e^{-2 c s} u(s) d s$ for $t \in\left[0, t_{1}\right]$ or $u(t)=e^{2 c t}\left(\frac{d w(t)}{d t}\right)$ for $t \in\left[0, t_{1}[\right.$ and $w\left(t_{1}\right)=0$. Next, taking the real part gives

$$
\begin{gathered}
\operatorname{Re} \int_{0}^{t_{1}} e^{2 c t}\left\{\left(\frac{d w}{d t}, w\right)-\left(\frac{d w}{d t}, c_{0} A_{0}^{*^{-1}}(t) w\right.\right. \\
\left.\left.+\frac{d A_{0}^{*^{-1}}(t)}{d t} w+A_{0}^{*^{-1}}(t) \frac{d w}{d t}\right)\right\} d t=0, \quad c \geq 0 .
\end{gathered}
$$

Here, the existence of a weak derivative $\frac{d A_{0}^{*^{-1}}(t)}{d t}=$ $\left(\frac{d A_{0}^{-1}(t)}{d t}\right)^{*} \in L_{\infty}\left(I_{r}, \mathscr{L}(H)\right)$ for the inverses $A_{0}^{*^{-1}}(t)$ of the operators $A_{0}^{*}(t)=A^{*}(t)+c_{0} I$ follows from Condition II. The first scalar product in (9) is integrated once by part with respect to $t$ and inequalities (2) and (4) and the obvious relation $\left[A_{0}^{-1}(t) g\right]_{(t)}=\left\langle A_{0}^{*^{-1}}(t) g\right\rangle_{(t)}$ are applied to the second scalar product to obtain

$$
\begin{gathered}
c \int_{0}^{t_{1}} e^{2 c t}|w|^{2} d t+\int_{0}^{t_{1}} e^{2 c t}\left\langle A_{0}^{*^{-1}}(t) \frac{d w}{d t}\right\rangle_{(t)}^{2} d t \\
-\left(c_{0} c_{1}^{-1 / 2}+c_{2}(0)\right) \int_{0}^{t_{1}} e^{2 c t}\left\langle A_{0}^{*^{-1}}(t) \frac{d w}{d t}\right\rangle_{(t)}|w| d t \leq 0 .
\end{gathered}
$$

For $c>\frac{\left(c_{0} c_{1}^{-1 / 2}+c_{2}(0)\right)^{2}}{4}$, combining this relation with the Cauchy-Schwarz inequality, we conclude that $w(t)=0$ and $u(t)=0$ for a.a. $t \in I_{0}$.

To show that $u=0$ almost everywhere on $I_{1}=\left[t_{1}, t_{2}[\right.$, we substitute into (8)

$$
\varphi(t)=\left\{\begin{array}{l}
0, \quad t_{2} \leq t \leq T \\
A_{0}^{*^{-1}}(t) w(t), \quad t_{1} \leq t<t_{2} \\
\psi_{0}(t), \quad 0 \leq t<t_{1},
\end{array}\right.
$$


where $w(t)=-\int_{t}^{t_{2}} e^{-2 c s} u(s) d s$ for $t \in\left[t_{1}, t_{2}[\right.$ or $u(t)=$ $e^{2 c t}\left(\frac{d w(t)}{d t}\right)$ for $t \in\left[t_{1}, t_{2}\left[\right.\right.$ and $w\left(t_{2}\right)=0$, while $\psi_{0}(t)$ is a weak solution to the reverse-time Cauchy problem

$$
\begin{gathered}
\frac{d \psi_{0}(t)}{d t}-B^{*}(t) \psi_{0}(t)=0, \quad 0<t<t_{1} ; \\
\psi_{0}\left(t_{1}\right)=v_{0,0}=A_{0}^{*-1}\left(t_{1}\right) w\left(t_{1}\right) .
\end{gathered}
$$

By the substitution $\tau=t_{1}-t$, this problem is reduced to the direct-time Cauchy problem

$$
\begin{gathered}
\frac{d \theta_{0}(\tau)}{d \tau}+B_{0}^{*}(\tau) \theta_{0}(\tau)=0, B_{0}^{*}(\tau)=B^{*}\left(t_{1}-\tau\right) \\
0<\tau<t_{1} ; \quad \theta_{0}(0)=V_{0,0}
\end{gathered}
$$

for the new function $\theta_{0}(\tau)=\psi_{0}(t)$.

For the first time, we prove the following smoothness theorem.

Theorem 2. Let Conditions III(a) and III(b) be satisfied.

Then, for any $f_{r} \in L_{2}\left(I_{r}, W^{*}(t)\right)$ and $v_{r, 0} \in W^{*}\left(t_{r}\right)$, the Cauchy problems

$$
\begin{gathered}
\frac{d v_{r}(\tau)}{d \tau}+B_{0}^{*}(\tau) v_{r}(\tau)=f_{r}(\tau), \\
\tau \in] t_{r}, t_{r+1}\left[; \quad v_{r}\left(t_{r}\right)=v_{r, 0}, \quad r=0,1, \ldots, R-1\right.
\end{gathered}
$$

have unique weak solutions $v_{r} \in \mathcal{H}_{r}=L_{2}\left(I_{r}, H\right)$ with the properties

$$
\begin{gathered}
V_{r}(\tau) \in D\left(B_{0}^{*}(\tau)\right), \quad \tau \in I_{r} ; \\
\frac{d^{k} V_{r}(\tau)}{d \tau^{k}} \in L_{2}\left(I_{r}, W^{* 1-k}(\tau)\right), \quad k=0,1, \\
k=0,1, \quad r=0,1, \ldots, R-1 .
\end{gathered}
$$

Here, the Hilbert space $W^{* 1}(T)$ is the domain $D\left(B_{0}^{*}(\tau)\right)$ of the operators $B_{0}^{*}(\tau)=B^{*}\left(t_{r+1}+t_{r}-\tau\right)$ with Hermitian norms $\|\nabla\|_{(\tau)}=\left|B_{0}^{*}(\tau) v\right|, \tau \in I_{r}$

Embedding (6) with $r=1$ in Condition III(c) implies that the initial function $V_{0,0}$ in Cauchy problem (11) belongs to $\left.D B_{0}^{*}(0)\right)$. To this Cauchy problem, we apply Theorem 2 with $f_{r}=0, v_{r, 0}=v_{0,0}$, and $r=0$. As a result, we conclude that its weak solution satisfies $\theta_{0}(\tau) \in$ $D\left(B_{0}^{*}(\tau)\right)$ for $\tau \in I_{0}$ and $\frac{d \theta_{0}(\tau)}{d \tau}, B_{0}^{*}(\tau) \theta_{0}(\tau) \in L_{2}\left(I_{0}\right.$, $H)$. Therefore, $\varphi(t)$ given by (10) belongs to the set $\Phi$. Substituting this $\varphi(t)$ into (8) and taking the real part yields

DOKLADY MATHEMATICS Vol. $78 \quad$ No. 32008

$$
\begin{gathered}
\operatorname{Re}_{t_{1}}^{t_{2}} e^{2 c t}\left\{\left(\frac{d w}{d t}, w\right)-\left(\frac{d w}{d t}, c_{0} A_{0}^{*^{-1}}(t) w+\frac{d A_{0}^{*^{-1}}}{d t} w\right.\right. \\
\left.\left.+A_{0}^{*^{-1}}(t) \frac{d w}{d t}\right)\right\} d t=0, \quad c \geq 0
\end{gathered}
$$

Proceeding as before, we find that $u(t)=0$ for a.a. $t \in$ $I_{1}$, etc., with the only difference being that the functions $\varphi(t)=\eta_{r}(t) h_{r}(t)$ are substituted into (8) on the intervals $I_{r, r}=2,3, \ldots, R$. Here,

$$
\begin{aligned}
& h_{r}(t)=\left\{\begin{array}{l}
0, \quad t_{r+1} \leq t \leq T \\
A_{0}^{*^{-1}}(t) w(t), \quad t_{r} \leq t<t_{r+1} \\
\psi_{r-1}(t), \quad t_{r-1} \leq t<t_{r} \\
1, \quad 0 \leq t<t_{r-1},
\end{array}\right. \\
& w(t)=-\int_{t}^{t_{r+1}} e^{-2 c s} u(s) d s, \quad t \in I_{r} ;
\end{aligned}
$$

$\psi_{r-1}(t)$ are weak solutions to the reverse-time Cauchy problem

$$
\begin{gathered}
\frac{d \psi_{r-1}(t)}{d t}-B^{*}(t) \psi_{r-1}(t)=0, \quad t_{r-1}<t<t_{r} \\
\psi_{r-1}\left(t_{r}\right)=v_{r-1,0}=A_{0}^{*^{-1}}\left(t_{r}\right) w\left(t_{r}\right)
\end{gathered}
$$

and the cutoff functions $\eta_{r}(t) \in C^{\infty}(\mathbb{R})$ are defined as

$$
\eta_{r}(t)=\left\{\begin{array}{l}
0 \text { for } t \in\left[0, t_{r-1}\right] \\
1 \text { for } t \in\left[t_{r}, T\right]
\end{array}\right.
$$

$r=2,3, \ldots, R$. As a result, after $R+1$ steps, if $R<+\infty$, we conclude that $u(t)=0$ for a.a. $t \in I_{r}, r=0,1, \ldots, R$. The case $R=+\infty$ is reduced to $R<+\infty$ by using the concept of a complete measure in $] 0, T[$, since weak solutions to Cauchy problem (1) exist on almost the entire interval ]0, T[ without Conditions II and III.

Corollary 1. If Conditions I-III are satisfied, then, for any $f \in \mathscr{H}^{*-}$ and $u_{0} \in H$, the weak solutions $u \in \mathscr{H}$ to Cauchy problem (1) satisfy

$$
\int_{0}^{T}|u(t)|^{2} d t \leq \frac{4}{c_{1}}\left(\int_{0}^{T}\langle f(t)\rangle_{(-t)}^{2} d t+\left|u_{0}\right|^{2}\right)
$$

$\langle\cdot\rangle_{(-t)}$ is the norm in $H_{t}^{*^{-}}$.

Corollary 2. Suppose that Conditions I and II are satisfied and inequality (5) holds for $B(t)=A_{0}^{*}(t)$. Then, by Theorem 2, for any $f \in L_{2}\left(I_{r}, W(t)\right)$ and $u\left(t_{r}\right) \in$ $W\left(t_{r}\right)$, where $W(t)$ are the sets $D(A(t))$ equipped with the 
graph norm of the operators $A_{0}(t)$, the weak solutions to Cauchy problem (1) on $I_{r}$ are smooth; i.e.,

$$
\begin{gathered}
u(t) \in D(A(t)), \quad t \in I_{r} \\
\frac{d u(t)}{d t}, \quad A(t) u(t) \in L_{2}\left(I_{r}, H\right), \quad r=0,1, \ldots, R .
\end{gathered}
$$

Using the results of [5], we analyzed the well-posedness of the following mixed problems.

3. In a bounded domain $G=] 0, T[\times] 0, l[$, consider the equation

$$
\frac{\partial u(t, x)}{\partial t}+A(t) u(t, x)=f(t, x), \quad\{t, x\} \in G,
$$

where $A(t)=a(t) \frac{\partial^{3}}{\partial x^{3}}$ for $t \in\left[0, t_{1}\left[\right.\right.$ and $A(t)=-a(t) \frac{\partial^{6}}{\partial x^{6}}$ for $t \in\left[t_{1}, T\right]$, with the $t$-dependent boundary conditions

$$
\begin{gathered}
\frac{\partial^{2} u(t, 0)}{\partial x^{2}}=-a_{1}(t) u(t, 0), \\
\frac{\partial^{2} u(t, l)}{\partial x^{2}}=a_{2}(t) u(t, l), \quad \frac{\partial u(t, l)}{\partial x}=0, \\
\frac{\partial^{5} u(t, 0)}{\partial x^{5}}=-a_{1}(t) \frac{\partial^{3} u(t, 0)}{\partial x^{3}}, \\
\frac{\partial^{2} u(t, 0)}{\partial x^{2}}=a_{1}(t) u(t, 0), \quad \frac{\partial u(t, 0)}{\partial x}=0, \\
\frac{\partial^{5} u(t, l)}{\partial x^{5}}=a_{2}(t) \frac{\partial^{3} u(t, l)}{\partial x^{3}}, \\
\frac{\partial^{2} u(t, l)}{\partial x^{2}}=-a_{2}(t) u(t, l), \quad \frac{\partial^{4} u(t, l)}{\partial x^{4}}=0
\end{gathered}
$$

on the intervals $\left[0, t_{1}\left[\right.\right.$ and $\left[t_{1}, T\right]$, respectively, and with the initial conditions

$$
u(0, x)=u_{0}(x), \quad 0<x<l .
$$

Theorem 3. Suppose that the coefficients in (12)(14) satisfy $0<a_{0} \leq a(t), t \in[0, T], 0<a_{i, 0} \leq a_{i}(t), t \in$ $\left[0, t_{1}\left[, a_{i}(t) \geq 0, t \in\left[t_{1}, T\right], a(t) \in C^{(1)}\left(I_{r}\right), a_{i}(t) \in C[0, T] \cap\right.\right.$ $C^{(1)}\left(I_{r}\right), I=1,2, r=0,1, \ldots$

Then, for every $f \in L_{2}(] 0, T\left[, W_{2, t}^{*-}(0, l)\right)$ and $u_{0} \in$ $L_{2}(0, l)$, mixed problem (12)-(15) has a unique weak solution $u \in L_{2}(G)$. Here, $W_{2, t}^{*-}(0, l)$ are the antiduals of the Hilbert spaces $W_{2, t}^{*+}(0, l)$, which are the closures of the corresponding sets $D\left(A^{*}(t)\right)$ with respect to the Hermitian norms $\langle v\rangle_{(t)}=\left(\operatorname{Re}\left(A_{0}^{*}(t) v, v\right)_{0}\right)^{1 / 2}$.
4. In the bounded domain $G$, consider the variableorder equation

$$
\begin{aligned}
& \frac{\partial u(t, x)}{\partial t}+A_{r}(t) u(t, x)=f(t, x), \\
& x \in] 0, l\left[, \quad t \in I_{r}, \quad r=1,2, \ldots,\right.
\end{aligned}
$$

where

$$
\begin{gathered}
A_{r}(t) u(x)=a_{r}(t)(-1)^{r+m_{r}} \frac{\partial^{2\left(r+m_{r}\right)} u(x)}{\partial x^{2\left(r+m_{r}\right)}}, \\
t \in I_{r}=\left[\frac{T(r-1)}{r}, \frac{T r}{r+1}[\right.
\end{gathered}
$$

on the infinite partition $\left[0, T\left[=\coprod_{r=1}^{\infty} I_{r}\left(m_{r}\right.\right.\right.$ is a nondecreasing integer number sequence), with the boundary conditions

$$
\begin{gathered}
\frac{\partial^{2 i+1} u(t, 0)}{\partial x^{2 i+1}}-a_{1}(t) \frac{\partial^{2 i} u(t, 0)}{\partial x^{2 i}}=0, \\
\frac{\partial^{2 i+1} u(t, l)}{\partial x^{2 i+1}}+a_{2}(t) \frac{\partial^{2 i} u(t, l)}{\partial x^{2 i}}=0, \quad t \in I_{r}, \\
i=0,1, \ldots, r+m_{r}-1, \quad r=1,2, \ldots
\end{gathered}
$$

Theorem 4. Suppose that the coefficients satisfy $a<$ $a_{r, 0} \leq a_{r}(t), t \in I_{r}, a_{i}(t) \geq 0, t \in[0, T], a_{r}(t) \in C^{(1)}\left(I_{r}\right)$, $a_{i}(t) \in C[0, T] \cap C^{(1)}\left(I_{r}\right), i=1,2 ; r=1,2, \ldots$.

Then, for every $f \in L_{2}\left(I_{r}, W_{2, t}^{-r-m_{r}}(0, l)\right), r=1,2, \ldots$, and $u_{0} \in L_{2}(0, l)$, mixed problem (16), (17), (15) has a unique weak solution $u \in L_{2}(G)$. Here, $W_{2, t}^{-r-m_{r}}(0, l)$ are the antiduals of the Hilbert spaces $W_{2, t}^{r+m_{r}}(0, l)$, which are the closures of the corresponding sets $D\left(A^{r}(t)\right)$ with respect to the Hermitian norms $\langle v\rangle_{(t)}=\left(A_{r}^{*}(t) v+v, v\right)_{0}^{1 / 2}$.

\section{ACKNOWLEDGMENTS}

We are grateful to Academician E.I. Moiseev and Professor N.I. Yurchuk for their interest in this work and encouragement.

\section{REFERENCES}

1. J.-L. Lions, Equations différentielles opérationnelles et problémes aux limites (Springer-Verlag, Berlin, 1961).

2. N. I. Yurchuk, Extended Abstract of Doctoral Dissertation in Mathematics and Physics (Moscow, 1981).

3. F. E. Lomovtsev, Extended Abstract of Doctoral Dissertation in Mathematics and Physics (Minsk, 2003).

4. F. E. Lomovtsev, Vestsi Nats. Akad. Nauk Belarusi, Ser. Fiz.-Mat., No. 2, 4-11 (2007).

5. F. E. Lomovtsev, Differ. Equations 42, 874-881 (2006) [Differ. Uravn. 42, 820-826 (2006)].

DOKLADY MATHEMATICS Vol. 78 No. 32008 